\title{
Bayesian Prediction of Exponentiated Weibull Distribution based on Progressive Type II Censoring
}

\author{
Jinhyouk Jung ${ }^{a}$, Younshik Chung ${ }^{1, b}$ \\ ${ }^{a}$ Department of Analytics, Diloitte Consulting Korea \\ ${ }^{b}$ Department of Statistics, Pusan National University
}

\begin{abstract}
Based on progressive Type II censored sampling which is an important method to obtain failure data in a lifetime study, we suggest a very general form of Bayesian prediction bounds from two parameters exponentiated Weibull distribution using the proper general prior density. For this, Markov chain Monte Carlo approach is considered and we also provide a simulation study.
\end{abstract}

Keywords: Bayesian prediction bounds, exponentiated Weibull distribution, Gibbs sampling, Metropolis-Hastings algorithm, progressive Type II censoring.

\section{Introduction}

Two-parameter Exponentiated Weibull(EW) distribution introduced by Mudholkar and Srivastava (1993) is a more realistic model for an analysis of a lifetime test than a model with monotone failure rates. Lifetime data often have bathtub shape or upside-down bathtub shape failure rates. EW distribution often has non-monotone failure rates; therefore, it is applied as a failure model in areas of reliability, quality control, duration and failure time modeling that make it useful to fit many types of data.

Progressive Type II censored sampling is an important method to obtain failure data in lifetime studies. Live units removed early on can be readily used in other tests that save costs to the experimenter; consequently, a compromise can be achieved between time consumption and the observation of some extreme values.

In practical scenarios, the lifetimes of test units may not be recordable exactly because the test terminates after a predetermined number of failures occur in order to save time or costs. Some test units may need to be removed (for various reasons) at different stages in the study; consequently, would lead to progressive censoring. Some early works can be found in Viveros and Balakrishnan (1994) and Balakrishnan and Sandhu (1995). Nassar and Eissa (2004) deal with complete and Type II censoring samples and develop the estimation of the shape parameters and reliability function of the exponentiated Weibull model under Type II Progressive censoring samples.

Under this censoring scheme, Kim et al. (2011) considered the Bayesian estimation of the parameters and the reliability function of the EW distribution with progressive Type II censored sampling. They obtained Bayes estimators using symmetric and asymmetric loss functions via squared error loss and Linex loss functions; in addition, they used an approximation based on the Lindley (1980) method to obtain Bayes estimates under the loss functions.

\footnotetext{
${ }^{1}$ Corresponding author: Professor, Department of Statistics, Pusan National University, Busan 609-735, Korea.

E-mail: yschung@pusan.ac.kr
} 
The main issue of this study is the prediction problems of the lifetime models. Problems arise when machine tools need to be replaced or industrial processes replaced in the field of engineering and the provision of warranty limits for the future performance of a specified number of systems in the field of business. This article is concerned with construction of Bayesian prediction bounds for a future progressive order statistic based on an informative Type II progressive censoring right censored sample from the exponentiated Weibull distribution. In Section 2, we provide a brief explanation for the progressive censoring and EW model. We then suggest Bayesian prediction bounds as a general form for two parameters EW distribution in Section 3. In Section 4, we present the results of simulation used in MCMC methods. The concluding remarks are in Section 5.

\section{Progressive Type II Censoring and EW Mpdel}

Suppose that $n$ independent items are put on a life test. They consist of $m$ ordered observed failure times denoted by $t_{1}, \ldots, t_{m}$ and progressive censoring scheme $\left(R_{1}, \ldots, R_{m}\right)$ which are previously fixed. It is clear that $n=m+R_{1}+R_{2}+\cdots+R_{m}$.

Note that the familiar complete and Type II right-censored samples are special cases of this scheme. If $R_{1}=R_{2}=\cdots=R_{m-1}=0$ and $R_{m}=n-m$, this sampling scheme reduces to the conventional Type II censoring. In addition, if $R_{1}=R_{2}=\cdots=R_{m}=0$, then the progressive Type II censoring scheme reduces to the complete sampling case.

Balakrishnan and Sandhu (1995) showed the joint probability density function is provided as: when the failure times of $n$ items originally on the test come from a continuous population with cumulative density function $F(t)$ (cdf) and probability density function $f(t)$ (pdf),

$$
P(t \mid \theta)=A \prod_{i=1}^{m} f\left(t_{i}\right)\left[1-F\left(t_{i}\right)\right]^{R_{i}},
$$

where $\theta$ is the parameter vector and

$$
A=n\left(n-R_{1}-1\right)\left(n-R_{1}-R_{2}-2\right) \cdots\left(n-R_{1}-R_{2}-\cdots-R_{m-1}-m+1\right) .
$$

Now we suppose that $T$ is a positive random variable having cdf $F(t)$ such as:

$$
F(t) \equiv F(t \mid \theta)=e^{-\lambda_{\theta}(t)}, \quad t>0,
$$

where $\theta$ is the parameter (could be a vector) that indicates the population distribution such that $\theta \in$ $\Theta, \Theta$ is the parameter space, and $\lambda_{\theta}(t) \equiv \lambda(t ; \theta)$. Notice that $\lambda_{\theta}(t)$ is assumed to be a nonnegative continuous differentiable function of $t$ such that $\lambda_{\theta}(t) \rightarrow \infty$ as $t \rightarrow 0^{+}$and $\lambda_{\theta}(t) \rightarrow 0$ as $t \rightarrow \infty$. Its corresponding pdf $f(t)$ and reliability function are given respectively as:

$$
f(t) \equiv f(t \mid \theta)=-\lambda_{\theta}^{\prime}(t) e^{-\lambda_{\theta}(t)}, \quad t>0,
$$

and

$$
S(t) \equiv S(t \mid \theta)=1-F(t)=e^{-\lambda_{\theta}(t)},
$$

where the prime in (2.3) indicates the first derivatives for the parameter. 
If we assume that the sample is a progressive Type II censored sample, equation in (2.1) can be rewritten as the likelihood function such as:

$$
L(\theta ; t) \propto \prod_{i=1}^{m} f\left(t_{i}\right)\left[S\left(t_{i}\right)\right]^{R_{i}} .
$$

Substituting (2.3) and (2.4) in (2.5), the likelihood function based on progressive Type II censored sample can be expressed as:

$$
L(\theta ; t) \propto\left(\prod_{i=1}^{m}\left(-\lambda_{\theta}^{\prime}\left(t_{i}\right)\right)\right) e^{-\sum_{i=1}^{m}\left[\lambda_{\theta}\left(t_{i}\right)-R_{i} \ln \left(1-e^{-\lambda_{\theta}\left(t_{i}\right)}\right)\right]} .
$$

To set up a Bayesian method, we consider the proper general (conjugate) prior density suggested by Al-Hussaini (1999). The form of which is given as:

$$
\pi(\theta ; z) \propto C(\theta ; z) e^{-D(\theta ; z)}, \quad \theta \in \Theta, z \in \Omega,
$$

where $z, C(\theta ; z), D(\theta ; z)$ and $\Omega$ are vector of prior parameters, parametric function, exponential powerterm and the hyper-parameter space, respectively. Then the posterior density function can be given by:

$$
\pi(\theta \mid t) \propto\left(\prod_{i=1}^{m}\left(-\lambda_{\theta}^{\prime}\left(t_{i}\right)\right)\right) C(\theta ; z) \times e^{-\sum_{i=1}^{m}\left[\lambda_{\theta}\left(t_{i}\right)-R_{i} \ln \left(1-e^{-\lambda_{\theta}\left(t_{i}\right)}\right)\right]-D(\theta ; z)} .
$$

Mudholkar and Srivastava (1993) introduced Two-parameter Exponentiated Weibull(EW) distribution with $\alpha$ and $\beta$. Cumulative density function and probability density function are given respectively by

$$
F(t)=\left(1-e^{-t^{\alpha}}\right)^{\beta}, \quad t>0
$$

and

$$
f(t)=\alpha \beta t^{\alpha-1} e^{-t^{\alpha}}\left(1-e^{-t^{\alpha}}\right)^{\beta-1}, \quad t>0, \alpha>0, \beta>0 .
$$

Now we consider a bivariate prior density suggested by Nassar and Eissa (2004). They suggested a bivariate prior density which is of form as:

$$
\pi(\alpha, \beta)=\pi_{2}(\beta \mid \alpha) \pi_{1}(\alpha)
$$

where

$$
\pi_{1}(\alpha)=\frac{1}{b} e^{-\frac{\alpha}{b}}
$$

and

$$
\pi_{2}(\beta \mid \alpha)=\frac{\alpha^{-v}}{\Gamma(v)} \beta^{\nu-1} e^{-\frac{\beta}{\alpha}}, \quad \beta>0
$$


Here, $b$ and $v$ are assumed to be known. Therefore, the bivariate prior density function of $\alpha$ and $\beta$ can be written as:

$$
\pi(\alpha, \beta)=(b \Gamma(v))^{-1} \alpha^{-v} \beta^{v-1} e^{-\frac{\alpha^{2}+b \beta}{b \alpha}}, \quad \alpha>0, \beta>0 .
$$

Now the joint posterior density function of $\alpha$ and $\beta$ given $t$ is proportional to

$$
\pi(\alpha, \beta \mid t) \propto \alpha^{m-v} \beta^{m+\nu-1} e^{-\left(\frac{\alpha}{b}+\varphi_{1}-\varphi_{2}+\frac{\beta}{\alpha}\right)},
$$

where $\varphi_{1}$ and $\varphi_{2}$ are:

$$
\begin{aligned}
\varphi_{1}(\alpha) & =\sum_{i=1}^{m}\left[t_{i}^{\alpha}-(\alpha-1) \ln t_{i}+\ln u_{i}\right], \\
\varphi_{2}(\alpha, \beta) & =\sum_{i=1}^{m}\left[\beta \ln u_{i}+R_{i} \ln \left(1-u_{i}^{\beta}\right)\right],
\end{aligned}
$$

and $u_{i}=\left(1-e^{-t_{i}^{\alpha}}\right)$

If we want to express general form such like (2.8) with (2.15), $\lambda(t ; \alpha, \beta), C(\alpha, \beta ; z), D(\alpha, \beta ; z)$ and $z=(v, b)$ are respectively:

$$
\begin{aligned}
\lambda(t ; \alpha, \beta) & =-\beta \ln \left(1-e^{-t^{\alpha}}\right), \\
C(\alpha, \beta ; v) & =\alpha^{-v} \beta^{\nu-1},
\end{aligned}
$$

and

$$
D(\alpha, \beta ; b)=\frac{\alpha}{b}+\frac{\beta}{\alpha}
$$

\section{Bayesian Predictive Bounds}

The general problem of statistical prediction may be described as that of inferring the value of unknown observable which belongs to a future sample from current available information, known as the informative sample.

In Bayesian prediction problems, we generally try to predict a random variable $Y \sim g(y \mid \theta)$ based on the observation of $T \sim f(t \mid \theta)$. We further assume that $T$ and $Y$ are independent and $g$ is a proper density. If $T$ and $Y$ are not independent, the necessary change in the following would be to replace $g(y \mid \theta)$ by $g(y \mid \theta, t)$. The idea of Bayesian predictive inference is that, since $\pi(\theta \mid t)$ is the believed (posterior) distribution of $\theta$, then $g(y \mid \theta) \pi(\theta \mid t)$ is the joint distribution of $y$ and $\theta$ given $t$, and integrating out over $\theta$ will provide the assumed distribution of $y$ given $t$. Therefore, the predictive density of $Y$ given vector $t$ is defined by:

$$
f(y \mid t)=\int_{\Theta} g(y \mid \theta) \pi(\theta \mid t) d \theta .
$$

Now, a $100 \tau^{*} \%$ Bayesian prediction bounds for $Y \equiv Y_{s}$ is such that $P\left[L_{s}(t) \leq Y_{s} \leq U_{s}(t)\right]=\tau^{*}$, where $L_{s}(t)$ and $U_{s}(t)$ are the lower and upper Bayesian predictive bounds of the $s^{\text {th }}$ order statistic 
$Y_{s}, s=1,2, \ldots, N$, which satisfy the following two equations:

$$
\begin{aligned}
& P_{r}\left(Y_{s} \geq L_{s}(t) \mid t\right)=\frac{1+\tau^{*}}{2}, \\
& P_{r}\left(Y_{s} \geq U_{s}(t) \mid t\right)=\frac{1-\tau^{*}}{2} .
\end{aligned}
$$

By using (3.1) and (3.2), we can find its predictive bounds.

This study presents the general forms of $g\left(y_{s} \mid \theta\right), f\left(y_{s} \mid t\right)$ and the probability when the future observation is larger than for any $\epsilon$, respectively. To do this, we assume that $T_{1}, \ldots, T_{m}$ as informative sample and $R_{1}, R_{2}, \ldots, R_{m}$ as a censoring scheme from a random sample of size $n$ and $Y_{1}, \ldots, Y_{N}$ as a future ordered sample of size $N$. If it is assumed that two samples are independent and each corresponding sample is obtained from a population with cdf (2.2), then the density function of $Y_{s}$, $s=1, \ldots, N$ is given by

$$
g\left(y_{s} \mid \theta\right)=s\left(\begin{array}{c}
N \\
s
\end{array}\right) \sum_{j=0}^{N-s}(-1)^{j}\left(\begin{array}{c}
N-s \\
j
\end{array}\right)\left(-\lambda_{\theta}^{\prime}\left(y_{s}\right)\right) e^{-(s+j) \lambda_{\theta}\left(y_{s}\right)} .
$$

The above expression can be obtained by using the formula of the $s^{\text {th }}$ general order statistic, by substituting (2.2) and (2.3), and by using a binomial theorem. We calculate the predictive density function $f\left(y_{s} \mid t\right)$ from the posterior density function (2.8) and density function (3.3). It follows that:

$$
f\left(y_{s} \mid t\right)=s\left(\begin{array}{c}
N \\
s
\end{array}\right) \sum_{j=0}^{N-s}(-1)^{j}\left(\begin{array}{c}
N-s \\
j
\end{array}\right) \xi_{j}\left(y_{s}\right)
$$

where

$$
\begin{aligned}
\xi_{j}\left(y_{s}\right) & =\int_{\Theta} \eta(\theta) e^{-\Psi(\theta)} d \theta, \\
\eta(\theta) & =\left(-\lambda_{\theta}^{\prime}\left(y_{s}\right)\right)\left(\prod_{i=1}^{m}\left(-\lambda_{\theta}^{\prime}\left(t_{i}\right)\right)\right) C(\theta ; z),
\end{aligned}
$$

and

$$
\Psi(\theta)=\sum_{i=1}^{m}\left[\lambda_{\theta}\left(t_{i}\right)+R_{i} \ln \left(1-e^{-\lambda_{\theta}\left(t_{i}\right)}\right)\right]+D(\theta ; z)+(s+j) \lambda_{\theta}\left(y_{s}\right) .
$$

Now, we calculate the predictive probability for an arbitrary $\epsilon>0$. If the predictive density function $f\left(y_{s} \mid t\right)$ is represented by (3.4) then the predictive probability of the $s^{\text {th }}$ future observation greater than for the arbitrary $\epsilon>0$ can be calculated as

$$
\begin{aligned}
P\left[Y_{s}>\epsilon \mid t\right] & =\int_{\epsilon}^{\infty} f\left(y_{s} \mid t\right) d y_{s} \\
& =s\left(\begin{array}{c}
N \\
s
\end{array}\right) \sum_{j=0}^{N-s} w_{j} G_{j}(\epsilon),
\end{aligned}
$$


where

$$
\begin{aligned}
w_{j} & =\frac{(-1)^{j}\left(\begin{array}{c}
N-s \\
j
\end{array}\right)}{s+j}, \\
G_{j}(\epsilon) & =\int_{\Theta}\left[1-e^{-(s+j) \lambda_{\theta}(\epsilon)}\right] \pi(\theta \mid t) d \theta .
\end{aligned}
$$

To show (3.5) using the predictive function in (3.4), we perform an integration from $\epsilon$ to $\infty$. We then obtain the following form:

$$
\begin{aligned}
P\left[Y_{s}>\epsilon \mid t\right] & =\int_{\epsilon}^{\infty} \int_{\Theta} g\left(y_{s} \mid \theta\right) \pi(\theta \mid t) d \theta d y_{s} \\
& =s\left(\begin{array}{c}
N \\
s
\end{array}\right) \sum_{j=0}^{N-s}(-1)^{j}\left(\begin{array}{c}
N-s \\
j
\end{array}\right) \times \int_{\Theta} \pi(\theta \mid t)\left(\int_{\epsilon}^{\infty}\left(-\lambda_{\theta}^{\prime}\left(y_{s}\right)\right) e^{-(s+j) \lambda_{\theta}\left(y_{s}\right)} d y_{s}\right) d \theta .
\end{aligned}
$$

Clearly as $y_{s} \rightarrow \infty$ then $\lambda_{\theta}\left(y_{s}\right) \rightarrow 0$ and as $y_{s} \rightarrow \epsilon$ then $\lambda_{\theta}\left(y_{s}\right) \rightarrow \lambda(\epsilon)$, and thus

$$
\int_{\epsilon}^{\infty}\left(-\lambda_{\theta}^{\prime}\left(y_{s}\right)\right) e^{-(j+s) \lambda_{\theta}\left(y_{s}\right)} d y_{s}=\frac{\left[1-e^{-(j+s) \lambda_{\theta}(\epsilon)}\right]}{(j+s)} .
$$

Therefore it can be rewritten that

$$
P\left[Y_{s}>\epsilon \mid t\right]=s\left(\begin{array}{c}
N \\
s
\end{array}\right) \sum_{j=0}^{N-s} \frac{(-1)^{j}\left(\begin{array}{c}
N-s \\
j
\end{array}\right)}{(j+s)} \int_{\Theta}\left[1-e^{-(j+s) \lambda_{\theta}(\epsilon)}\right] \pi(\theta \mid t) d \theta .
$$

Notice that $\lambda_{\theta}\left(y_{s}\right)$ would be changed to $\lambda_{\theta}(\epsilon)$ in (3.6). It does not lead to a simple form, but requires numerical integration, programming and computer time. Thus a simple approximation via an asymptotic expansion of non-tractable integral is considered and shown in the last part in this section.

A bivariate prior density suggested by Nassar and Eissa (2004) was adopted for a special case of the proper general prior density suggested by Al-Hussaini (1999). The Bayes predictive density function of $Y \equiv Y_{s}$ of (3.4) by applying the full conditional posterior density function of $\alpha$ and $\beta$ of (2.15) to this. We then can obtain the following form.

$$
\begin{aligned}
f\left(y_{s} \mid t\right)= & \int_{0}^{\infty} s\left(\begin{array}{l}
N \\
s
\end{array}\right) \sum_{j=0}^{N-s} w_{j}\left[\frac{\alpha y_{s}^{\alpha-1} e^{-y_{s}^{\alpha}}}{1-e^{-y_{s}^{\alpha}}}\right] \alpha^{m-v} e^{\left[-\frac{\alpha}{b}-\varphi_{1}(\alpha)\right]} \\
& \times \int_{0}^{\infty} \beta^{m+v} e^{\left[\varphi_{2}(\alpha, \beta)-\frac{\beta}{\alpha}+\beta(s+j) \ln \left(1-e^{-y_{s}^{\alpha}}\right)\right]} d \beta d \alpha .
\end{aligned}
$$

Finally the Bayesian prediction bounds for $Y \equiv Y_{s}$ are obtained by integrating out $y_{s}$. In order to get $P\left(Y_{s} \geq \epsilon \mid t\right)$ for some given value of $\epsilon$, we adopted Markov chain Monte Carlo(MCMC) methods such as Gibbs sampler (Gelfand and Smith, 1990) and Metropolis-Hastings algorithm (Metropolis et al, 1953; Hastings, 1970).

For using MCMC method, we need to compute a full conditional posterior density such as $\pi(\alpha \mid \beta, t)$ 
and $\pi(\beta \mid \alpha, t)$.

$$
\begin{aligned}
\pi(\alpha \mid \beta, t) \propto & \alpha^{m-v} \exp \left[-\frac{\alpha}{b}-\varphi_{1}(\alpha)+\varphi_{2}(\alpha, \beta)-\frac{\beta}{\alpha}\right] \\
\propto & \operatorname{Gam}\left(m-v+1,\left(\frac{1}{b}\right)^{-1}\right) \\
& \times \exp \left(-\frac{\beta}{\alpha}-\varphi_{1}(\alpha)+\varphi_{2}(\alpha, \beta)\right),
\end{aligned}
$$

where $\varphi_{1}$ and $\varphi_{2}$ are given by $(2.16)$, and $\operatorname{Gam}(\cdot, \cdot)$ indicates a gamma density function used for the deriving function. In addition, the conditional posterior density of $\pi(\beta \mid \alpha, t)$ is:

$$
\begin{aligned}
\pi(\beta \mid \alpha, t) \propto & \beta^{m+\nu-1} \exp \left[\varphi_{2}(\alpha, \beta)-\frac{\beta}{\alpha}\right] \\
\propto & \operatorname{Gam}\left(m+v,\left(\frac{1}{\alpha}-\sum_{i=1}^{m} \ln u_{i}\right)^{-1}\right) \\
& \times \exp \left(\sum_{i=1}^{m} R_{i} \ln \left(1-u_{i}^{\beta}\right)\right),
\end{aligned}
$$

where $\varphi_{2}$ and $u_{i}$ are given by (2.16).

Then given $\alpha^{(k)}$ and $\beta^{(k)}$ as the $k^{\text {th }}$ iterate state, draw candidate $\alpha^{(*)}$ from the deriving function $\operatorname{Gam}\left(m-v+1,(1 / b)^{-1}\right)$ and draw candidate $\beta^{(*)}$ from $\operatorname{Gam}\left(m+v,\left(1 / \alpha-\sum_{i=1}^{m} \ln u_{i}\right)^{-1}\right)$, respectively, and accept $\alpha^{(*)}$ as $(k+1)^{t h}$ iterate state of $\alpha$ with acceptance probability

$$
\psi_{1}=\min \left(\frac{\exp \left(-\frac{\beta}{\alpha^{(*)}}-\hbar\left(\alpha^{(*)}\right)\right)}{\exp \left(-\frac{\beta}{\alpha^{(k)}}-\hbar\left(\alpha^{(k)}\right)\right)}, 1\right)
$$

where

$$
\hbar\left(\alpha^{*}\right)=\sum_{i=1}^{m}\left\{t_{i}^{\alpha^{(*)}}+\left(\alpha^{(*)}-1\right) \ln t_{i}+\ln u_{i}^{(1-\beta)}-R_{i} \ln \left(1-u_{i}^{\beta}\right)\right\},
$$

and

$$
\hbar\left(\alpha^{(k)}\right)=\sum_{i=1}^{m}\left\{t_{i}^{\alpha^{(k)}}+\left(\alpha^{(k)}-1\right) \ln t_{i}+\ln u_{i}^{(1-\beta)}-R_{i} \ln \left(1-u_{i}^{\beta}\right)\right\} .
$$

By same method, $\beta^{(*)}$ as $(k+1)^{t h}$ iterate state of $\beta$ with acceptance probability

$$
\psi_{2}=\min \left(\frac{\exp \left(\sum_{i=1}^{m} R_{i} \ln \left(1-u_{i}^{\beta^{(*)}}\right)\right)}{\exp \left(\sum_{i=1}^{m} R_{i} \ln \left(1-u_{i}^{\beta^{(k)}}\right)\right)}, 1\right) .
$$


MLE will be used as initial value in this situation. Using the $\alpha$ and $\beta$ obtained by (3.10) and (3.11), the predictive probability of $y_{s}$ to be greater than for any $\epsilon$ is rewritten as:

$$
\begin{aligned}
\operatorname{Pr}\left(Y_{s} \geq \epsilon \mid t\right) & =\int_{\epsilon}^{\infty} f\left(y_{s} \mid t\right) d y_{s} \\
& =\int_{\epsilon}^{\infty}\left[\int_{0}^{\infty} \int_{0}^{\infty} g\left(y_{s} \mid \alpha, \beta\right) \pi(\alpha, \beta \mid t) d \alpha d \beta\right] d y_{s} \\
& \simeq \int_{\epsilon}^{\infty} \frac{1}{K} \sum_{k=1}^{K} g\left(y_{s} \mid \alpha^{(k)}, \beta^{(k)}\right) d y_{s} \\
& \simeq \frac{1}{K} \sum_{k=1}^{K} s\left(\begin{array}{c}
N \\
s
\end{array}\right) \sum_{j=0}^{N-s} w_{j}\left[1-\left(1-e^{-\epsilon^{(k)}}\right)^{\beta^{(k)}(s+j)}\right],
\end{aligned}
$$

where $w_{j}$ is given by (3.6), $\alpha^{(k)}$ and $\beta^{(k)}$ are outputs obtained by after $k$ Gibbs iteration.

We present our simulation results for 4 kinds of different censoring scheme in the next section.

\section{Simulation Study}

For our simulation study, we generated a progressive Type II censored sample from the EW distribution using the algorithm of Balakrishnan and Sandhu (1995). For a given value of $b, v$ and the progressive censoring scheme $R_{1}, R_{2}, \ldots, R_{m}$, we generated $\alpha$ and $\beta$ by (2.12) and (2.13), respectively. Next, we generated $Z_{1}, Z_{2}, \ldots, Z_{m}$ from $U(0,1)$ and $Q_{i}=Z_{i}^{1 /\left(i+R_{m}+R_{m-1}+\cdots+R_{m-i+1}\right)}$, for $i=1,2, \ldots, m$. We also made $U_{i}=1-Q_{m} Q_{m-1} \cdots Q_{m-i+1}$ which would be a progressive Type II censored sample of size $m$ from $U(0,1)$. Finally, we obtained a censored sample size $m$ from the EW distribution using the inverse cdf,

$$
T_{i}=\left[-\ln \left(1-U_{i}^{\frac{1}{\beta}}\right)\right]^{\frac{1}{\alpha}}, \quad i=1,2, \ldots, m .
$$

They are the required progressive Type II censored sample of size $m$ from EW distribution. By the above simulation algorithm, we obtained data $T(i)$, under the progressive Type II censoring schemes of four kinds which are $R 1=(1,0,1,2,0,0,3,0,1,2), R 2=(0,0,0,0,0,0,0,0,0,10)$, $R 3=(1,1,1,1,1,1,1,1,1,1)$ and $R 4=(2,2,2,2,2,0,0,0,0,0)$.

Our simulation setting is: First, we generate sample size of $n=20$ and a simulated progressively Type-II censored of size $m=10$ from $\operatorname{EW}(\alpha, \beta)$ density given by (2.10). For using bivariate prior distribution such as (2.14), we set $b=4$ to generate $\alpha$ in (2.12) and set $v=3$ with a generated $\alpha$ to generate $\beta$ in (2.13). Once we have to decide the value of $\tau^{*}$ in (3.2). In this paper, we set $\tau^{*}=0.95$ since we want to know $95 \%$ predictive intervals then we know the probabilities that $Y_{s}$ is greater than lower bound $\left(L_{s}(t)\right)$ or upper bound $\left(U_{s}(t)\right)$ from (3.2) should be 0.975 and 0.025 , respectively. In fact, (3.2) and (3.12) are equivalent. Therefore, our target is to find the value of $\epsilon$ which is the same of lower bound or upper bound. and the value of $\epsilon$ in (3.12) will be changed by 0.0001 . These processes are continued until achieving differences between lower bound $\left(L_{s}(t)\right)$ and 0.975 or upper bound $\left(U_{s}(t)\right)$ and 0.025 will be less than 0.00001 .

We used (3.8) and (3.9) for Gibbs sampling. In addition, (3.10) and (3.11) are used to apply the Metropolis algorithm.

Table 1 through Table 4 show the predictive intervals for $Y_{s}, s=1, \ldots, 10$ for our four kinds of censoring scheme such as $R 1, R 2, R 3$ and $R 4$. For a visual understanding of our results we provide 
Table 1: The $95 \%$ predictive intervals for $Y_{s}$ under $R 1$

\begin{tabular}{cc|ccc}
\hline \hline$T_{i}$ & Censoring time & $Y_{s}$ & Lower bound & Upper bound \\
\hline$T_{1}$ & 0.9146 & $Y_{1}$ & 0.1001 & 1.0959 \\
$T_{2}$ & 1.0672 & $Y_{2}$ & 0.2407 & 1.1498 \\
$T_{3}$ & $Y_{3}$ & 0.3675 & 1.2026 \\
$T_{4}$ & 1.0998 & $Y_{4}$ & 0.4845 & 1.2743 \\
$T_{5}$ & 1.2538 & $Y_{5}$ & 0.6057 & 1.3910 \\
$T_{6}$ & 1.2547 & $Y_{6}$ & 0.7304 & 1.5604 \\
$T_{7}$ & 1.3979 & $Y_{7}$ & 0.8611 & 1.7795 \\
$T_{8}$ & 1.4301 & $Y_{8}$ & 1.0025 & 2.0718 \\
$T_{9}$ & 1.5635 & $Y_{9}$ & 1.1190 & 2.5104 \\
$T_{10}$ & 1.6526 & $Y_{10}$ & 1.1704 & 3.3986 \\
\hline \hline
\end{tabular}

Table 2: The 95\% predictive intervals for $Y_{s}$ under $R 2$

\begin{tabular}{cc|ccc}
\hline \hline$T_{i}$ & Censoring time & $Y_{s}$ & Lower bound & Upper bound \\
\hline$T_{1}$ & 0.9076 & $Y_{1}$ & 0.0511 & 1.0910 \\
$T_{2}$ & 1.0521 & $Y_{2}$ & 0.1536 & 1.1434 \\
$T_{3}$ & 1.0831 & $Y_{3}$ & 0.2634 & 1.1961 \\
$T_{4}$ & 1.2206 & $Y_{4}$ & 0.3798 & 1.2699 \\
$T_{5}$ & $Y_{5}$ & 0.5042 & 1.3939 \\
$T_{6}$ & 1.2213 & $Y_{6}$ & 0.6385 & 1.5726 \\
$T_{7}$ & 1.3278 & $Y_{7}$ & 0.7852 & 1.8082 \\
$T_{8}$ & 1.3513 & $Y_{8}$ & 0.9492 & 2.1338 \\
$T_{9}$ & 1.4082 & $Y_{9}$ & 1.1144 & 2.6433 \\
$T_{10}$ & 1.4420 & $Y_{10}$ & 1.1808 & 3.7236 \\
\hline \hline
\end{tabular}

Table 3: The 95\% predictive intervals for $Y_{s}$ under $R 3$

\begin{tabular}{cc|ccc}
\hline \hline$T_{i}$ & Censoring time & $Y_{s}$ & Lower bound & Upper bound \\
\hline$T_{1}$ & 0.9076 & $Y_{1}$ & 0.0935 & 1.0931 \\
$T_{2}$ & 1.0563 & $Y_{2}$ & 0.2302 & 1.1479 \\
$T_{3}$ & 1.0896 & $Y_{3}$ & 0.3562 & 1.2033 \\
$T_{4}$ & $Y_{4}$ & 0.4786 & 1.2778 \\
$T_{5}$ & 1.2422 & $Y_{5}$ & 0.6013 & 1.3929 \\
$T_{6}$ & 1.2430 & $Y_{6}$ & 0.7273 & 1.5537 \\
$T_{7}$ & 1.3797 & $Y_{7}$ & 0.8596 & 1.7675 \\
$T_{8}$ & 1.4136 & $Y_{8}$ & 1.0028 & 2.0590 \\
$T_{9}$ & 1.5105 & $Y_{9}$ & 1.1190 & 2.5104 \\
$T_{10}$ & 1.5854 & $Y_{10}$ & 1.1775 & 3.4099 \\
\hline \hline
\end{tabular}

Figure 1 corresponding to Table 1 and Table 2 as well as Figure 2 corresponding to Table 3 and Table 4 , respectively.

For the censoring scheme of $R 1=(1,0,1,2,0,0,3,0,1,2)$, we randomly removed $R_{i}$ at each censoring time point without any regulation. The censoring scheme of $R 2=(0,0,0,0,0,0,0,0,0,10)$ is a conventional Type II censoring (where we assigned all to the last of $R_{10}$ at the last censoring time point of $t_{10}$ ) is just to compare the results of any other progressive Type II censoring schemes. $R 3=(1,1,1,1,1,1,1,1,1,1)$ was intended to check how the result varies when all of $R_{i}$ has been distributed equally. Finally, we considered circumstance such as $R 4=(2,2,2,2,2,0,0,0,0,0)$.

Table 1 and Table 3 show that the Bayesian predictive bounds are very similar to each other. Even though we used different censoring scheme, the values of censoring time are also similar. The conventional Type II censoring scheme of $R 2$ show that the censoring time for $T_{8}$ through $T_{10}$ are very close; however, the corresponding Bayesian predictive bounds are still similar to $R 1$ and $R 2$.

Table 4 show us that the bandwidths of predictive intervals of $Y_{s}$ are shorter and are different than 
Table 4: The 95\% predictive intervals for $Y_{s}$ under $R 4$

\begin{tabular}{cc|ccc}
\hline \hline$T_{i}$ & Censoring time & $Y_{s}$ & Lower bound & Upper bound \\
\hline$T_{1}$ & 0.9076 & $Y_{1}$ & 0.5273 & 1.1005 \\
$T_{2}$ & 1.0609 & $Y_{2}$ & 0.7010 & 1.1508 \\
$T_{3}$ & 1.0971 & $Y_{3}$ & 0.8099 & 1.2017 \\
$T_{4}$ & $Y_{4}$ & 0.8917 & 1.2599 \\
$T_{5}$ & $Y_{5}$ & 0.9655 & 1.3286 \\
$T_{6}$ & 1.2709 & $Y_{6}$ & 1.0262 & 1.4093 \\
$T_{7}$ & $Y_{7}$ & 1.0711 & 1.5053 \\
$T_{8}$ & 1.4858 & $Y_{8}$ & 1.0978 & 1.6251 \\
$T_{9}$ & 1.5358 & $Y_{9}$ & 1.1190 & 1.7900 \\
$T_{10}$ & 1.6777 & $Y_{10}$ & 1.1435 & 2.0818 \\
\hline \hline
\end{tabular}
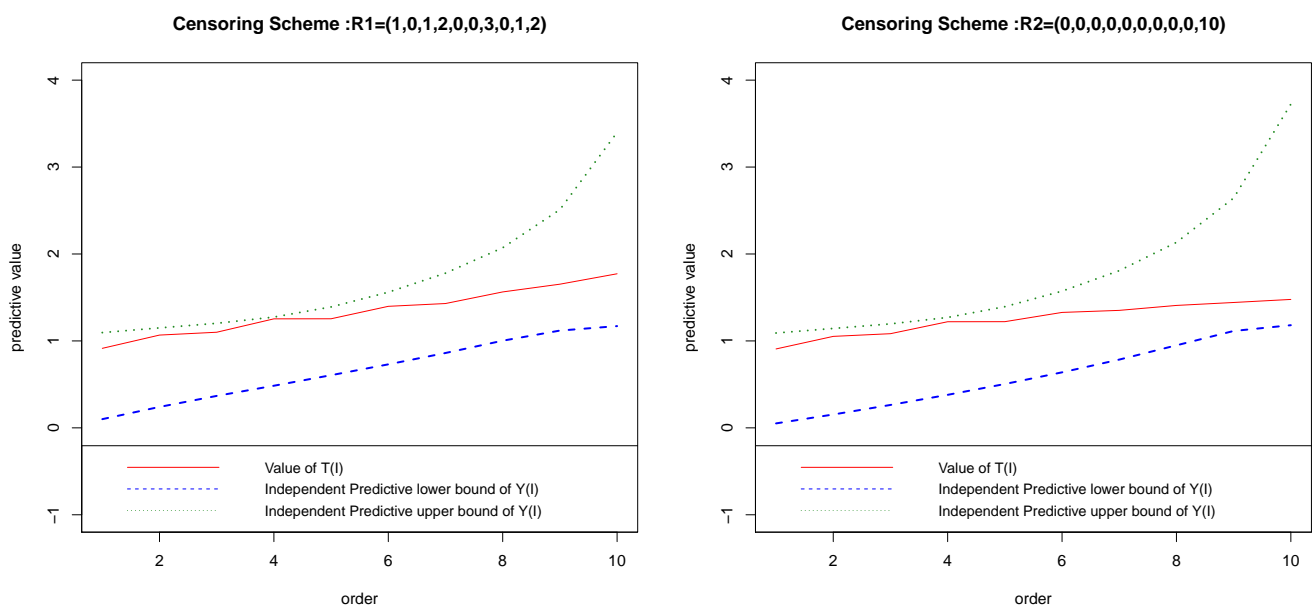

Figure 1: Plots for Predictive intervals of $Y_{s}$ under $R 1$ and $R 2$ censoring scheme
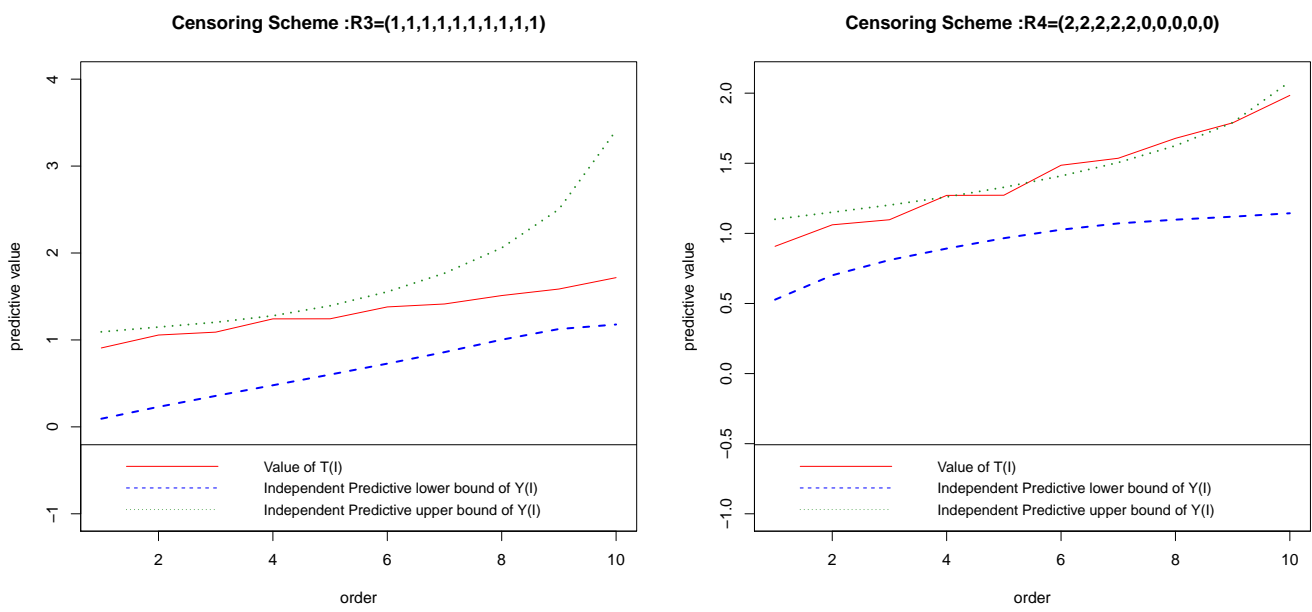

Figure 2: Plots for Predictive intervals of $Y_{s}$ under $R 3$ and $R 4$ censoring scheme

those of Table 1, Table 2, Table 3 and Table 4. In addition, the value of the predictive upper bounds run parallel to the value of the informative samples. We provide Figure 1 and Figure 2 to support 
Table 5: $95 \%$ predictive intervals for $Y_{s}$ as varying the hyperparameters

\begin{tabular}{|c|c|c|c|c|c|c|c|c|}
\hline \multirow{2}{*}{$Y_{s}$} & $b=2$ & $v=2$ & $b=6$ & $v=6$ & $b=7$ & $v=7$ & $b=8$ & $v=8$ \\
\hline & Lower & Upper & Lower & Upper & Lower & Upper & Lower & Upper \\
\hline$Y_{1}$ & 0.7122 & 1.2207 & 0.3153 & 1.3152 & 0.2203 & 1.3893 & 0.1602 & 1.4638 \\
\hline$Y_{2}$ & 0.8759 & 1.2973 & 0.5663 & 1.5357 & 0.4675 & 1.6999 & 0.3972 & 1.8681 \\
\hline$Y_{3}$ & 0.9714 & 1.3679 & 0.7559 & 1.7571 & 0.6767 & 2.0230 & 0.6196 & 2.3024 \\
\hline$Y_{4}$ & 1.0420 & 1.4366 & 0.9134 & 2.0002 & 0.8630 & 2.3913 & 0.8294 & 2.8115 \\
\hline$Y_{5}$ & 1.1001 & 1.5089 & 1.0467 & 2.2800 & 1.0301 & 2.8310 & 1.0247 & 3.4364 \\
\hline$Y_{6}$ & 1.1501 & 1.5884 & 1.1587 & 2.6185 & 1.1767 & 3.3773 & 1.2048 & 4.2386 \\
\hline$Y_{7}$ & 1.1961 & 1.6801 & 1.2543 & 3.0470 & 1.3082 & 4.0913 & 1.3708 & 5.3281 \\
\hline$Y_{8}$ & 1.2413 & 1.7934 & 1.3416 & 3.6317 & 1.4336 & 5.1007 & 1.5347 & 6.9366 \\
\hline$Y_{9}$ & 1.2896 & 1.9505 & 1.4329 & 4.5401 & 1.5682 & 6.7455 & 1.7154 & 9.7001 \\
\hline$Y_{10}$ & 1.3522 & 2.2398 & 1.5529 & 6.4775 & 1.7488 & 10.5400 & 1.9638 & 16.5628 \\
\hline
\end{tabular}

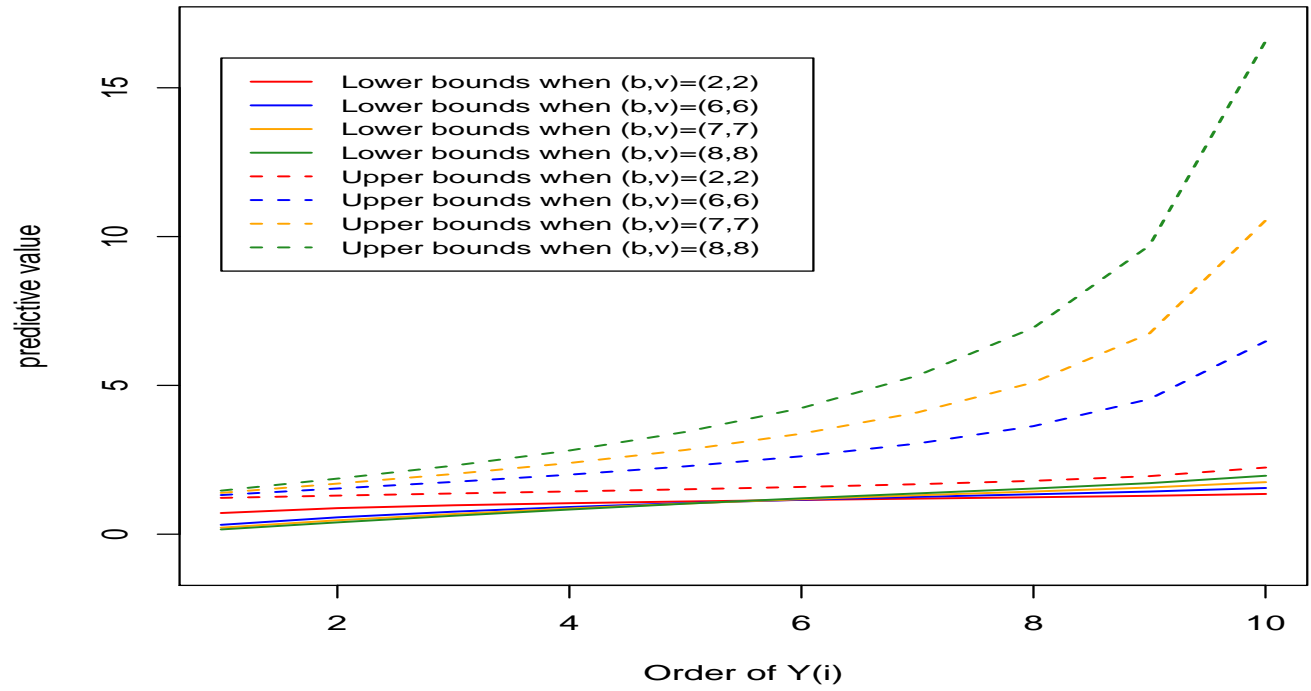

Figure 3: Plots for 95\% Predictive intervals of $Y_{s}$ under $R 1$

a more comprehensive understanding. Real line (red line), dotted line (green line) and dashed line (blue line) indicate the censoring time, upper bound and lower bound, respectively. We can find the differences among censoring schemes clearly.

Table 5 and Figure 3 shows how to change the result as varying the choice for hyper parameters of prior distribution. Under the censoring schemes of $R 1$, this simulation study was executed.

Table 5 illustrates how we set up the value of hyper-parameter of prior distribution such as $b$ and $v$ as $(2,2),(6,6),(7,7)$, and $(8,8)$, respectively. As the value of hyper-parameter goes to high, the bandwidths for the $95 \%$ predictive intervals are wider (see Figure 3 ).

\section{Concluding Remarks}

We show Bayesian prediction bounds for two parameters of Exponentiated Weibull distribution based on progressive Type II censoring. We consider an EW model and the above censoring scheme in a lifetime prediction problem in order to save application of data costs and time related to reliability and quality control. To present a general form of Bayesian predictive bounds to be calculated by numerical method, we first suggest a very general form for posterior density of EW model when using the 
proper general prior density and a simple approximation via an asymptotic expansion of non-tractable integral. Section 4 shows the four simulated difference censoring schemes; in addition, this trend was similar under censoring scheme $R 1, R 2$ and $R 3$ except for $R 4$. The bandwidth of prediction value for the upper and lower bound was shorter than the other censoring schemes for the censoring scheme of $R 4$. To the best of our knowledge, this is the first study on a general form of Bayesian predictive bounds in terms of an EW model with a progressive Type II censoring sample.

\section{References}

AL-Hussaini, E. K. (1999). Predicting observables from a general class of distribution, Journal of Statistical Planning and Inference, 79, 79-91.

Balakrishnan, N. and Sandhu, R. A. (1995). A simple simulation algorithm for generating progressive Type II censored samples, The American Statistician, 49, 229-230.

Gelfand, A. E. and Smith, A. F. M. (1990). Sampling-based approaches to calculating marginal densities, Journal of the American Statistical Association, 85, 398-409.

Hastings, W. K. (1970). Monte Carlo sampling methods using Markov chains and their applications, Biometrika, 57, 97-109.

Kim, C., Jung, J. and Chung, Y. (2011). Bayesian estimation for the exponentiated Weibull model under Type II progressive censoring, Statistical Papers, 52, 53-70.

Lindley, D. V. (1980). Approximate Bayesian methods, Trabajos de Stadistca, 21, 223-237.

Metropolis, N., Rosenbluth, A. W., Rosenbluth, M. N., Teller, A. H. and Teller, E. (1953). Equations of state calculations by fast computing machines, Journal of Chemical Physics, 21, 1087-1092.

Mudholkar, G. S. and Srivastava, D. K. (1993). Exponentiated Weibull family for analyzing bathtub failure-rate data, IEEE Transactions on Reliability, R-42,299-302.

Nassar, M. M. and Eissa, F. H. (2004). Bayesian Estimation for the Exponentiated Weibull Model, Communications in Statistics: Theory and Methods, 33, 2343-2362.

Viveros, R. and Balakrishnan, N. (1994). Interval estimation of life characteristics from progressively Censored data, Technometrics, 36, 84-91. 